\title{
Automatic Technique for Measuring The Electro-Mechanical Characteristics Of Quantum Tunneling Composites (QTC)
}

\author{
M.M. El-Tonsy \\ Physics Department, \\ Faculty of Science \\ Mansoura University \\ Mansoura, Egypt
}

\author{
H.A. Shaaban
}

Physics Department,

Faculty of Science

Mansoura University

Mansoura, Egypt

\author{
O.M.Al-Aqbi \\ R.M. Felfel \\ Ministry of education \\ Ministry of education \\ Baghdad-Iraq \\ Misrata - Libya
}

\begin{abstract}
Electrically conductive composites consisted of conducting fillers and the insulating polymer matrix. These composites could serve in preparation of piezoresistive composites (i.e. quantum tunnelling composites (QTC) due to their flexibility, light weight, easy processing, low cost, greater resistance changes, and ease of spreading over arbitrary curved surfaces.QTC have attracted tremendous attention due to their potential applications in advanced stress and strain sensors. Recently, various types of conducting materials and many soft polymers have been utilized in the manufacture of QTCs. Characterization of such composites involves several physical parameters. Therefore, a low cost technique was designed and manufactures in order to measure most of electro-mechanical properties of the produced composites with good accuracy and repeatability. QTCs were prepared by mixing poly-dimethyl siloxane (PDMS) and with different concentrations of graphite flakes (1:0.75, 1:1, 1:1.5, 1:1.75 and 1:2). This study declared the efficiency of the suggested technique as well as some fundamental features of the prepared composite. For example, conductivity of the composites containing higher concentration of graphite was found to be independent on rate of pressing during the test. It was also found that the capacitive behavior of the sample interrupted the flow of current at the instant of removing the applied pressure. The suggested setup has several advantages such as simplicity, high accuracy and providing lots of technical data that required for development and confirmation of models for the quantum tunnelling process.
\end{abstract}

Keywords: QTC, electro-mechanical, piezo-resistance, polymer/graphite composite, compressive capacitance, pressure sensors

\section{INTRODUCTION}

Traditional electro-conductive rubber is typically produced with vulcanized rubber filled with a high percentage of carbon black. Conductive particles of carbon, metals, carbon fibres, graphite, pyrolitic carbons and carbon black (with different purity and morphology) have been used as fillers in polymeric composites since the 50s [1]. Mechanism of electrical conduction in traditional polymeric composites is based on percolation. There is a critical threshold for the filler at which the sample suddenly becomes an excellent conductor as a result of formation of at the least one whole conductive path ensuring charge transport through the metallic particles. Beyond this threshold, the sample conductivity increases further since new paths formed by adding more metal filler until the saturation was obtained.

The conductivity of electro-conductive percolation-based composites commonly changes with any mechanical interactions. For instance, number of touching filler particles increases by applying compression pressure and consequently the number of conductive paths increases because of a higher relative concentration ratio.

The Quantum Tunnelling Composites (QTC) show similar behaviour to traditional metallic filled polymer composites, but www.ijsea.com conduction mechanism at the nano-scale is different. The conduction mechanism within QTC is based on quantum effects which makes them particularly suitable for specific applications. Two factors controlling the conductivity of the QTC;

- The presence of spiked metallic particles where the electrostatic fields strengthen and become able to conduct to another metal particle,

- The extreme wet ability (i.e. adhesion) between the metal particles and the polymeric matrix.

Within QTC, the conducting nano-particles will not touch even when the inter-particle distances diminished under compression. Therefore, classical conduction via percolation does not be valid. However, the charge transfer between the particles would be occurred through tunnelling at the spikes due to the decreased distances and extreme voltages.

QTC has extremely large and reversible changes in the electrical conductivity when twisted, bent, stretched, or compressed. This advantage would facilitate use of QTC in 
development of very simple and highly effective sensing systems such as tactile sensors $[2,3]$.

It is easy to predict the great efforts in future for studying, developing and producing more advanced QTC. Hence, the current work presents an automatic tool for direct dynamic measurement of some important physical quantities for QTC such as;

a) sample current as function of applied ascending and descending pressure,

b) sample resistance against applied pressure,

c) sample current and sample resistance as function of the pressing rate,

d) effect of viscoelastic relaxation properties of the host polymer matrix on the decay of sample resistance,

e) effect of curing period and temperature on sample conductivity at certain mechanical deformation,

f) influence of composition (matrix/filler ratio) on the conductivity of composites,

g) effect of sample thickness on the current and resistance at specific mechanical deformation,

h) impact of multi-component composite (i.e. contains several conductive fillers) on the sample conductivity at mechanical deformation,

i) influence of geometry of metallic particles on the conductivity against mechanical deformation.

Furthermore, many other physical parameters can be measured with reasonable accuracy and in short time. The suggested measuring setup could be easily modified by adding more options for wider ranges of measurements such as temperature and external magnetic field (Hall Effect) effects.

\section{SET UP OF THE SUGGESTED INSTRUMENT}

Figure 1 shows construction of the instrument.

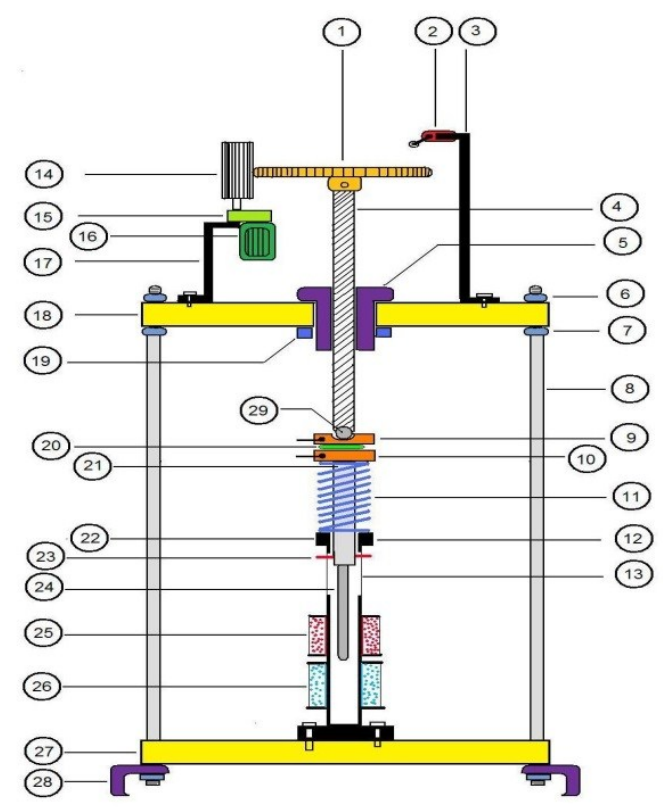

Figure 1: Schematic diagram of the measuring tool for characterising the physical properties of quantum tunnelling composites.

\begin{tabular}{|c|c|}
\hline 1 - Gear wheel & 2 - Automatic level switch \\
\hline 3 - Switch holder & $4-$ Screwed metallic rod \\
\hline 5 - Cylindrical nut with groove & $6 \& 7-$ Nuts \\
\hline 8 - Thick steel rod & 9 - Upper $\mathrm{Cu}$ disk electrode \\
\hline 10 - Lower $\mathrm{Cu}$ disk electrode & 11 - Stiff steel spring \\
\hline 12 or 22 - Copper cylinder & 13 - Two parallel slim windows \\
\hline 14 - Long slim gear & $15-$ Gear box \\
\hline $16-\mathrm{DC}$ motor & 17 - Motor holder \\
\hline 18 - Top plate (Teflon) & $19-$ Big nut \\
\hline $20-$ Sample & 21 - Copper rod \\
\hline 23 - Steel tip stopper & 24 - Iron rod \\
\hline 25 - Primary coil & $26-$ Secondary coils \\
\hline 27 - Base plate (Teflon) & 28 - Hard rubber feet \\
\hline
\end{tabular}

\section{WORKING MECHANISM OF THE INSTRUMENT:}

At the initial position, the gear wheel (1) is located at the most upper end of the long slim gear (14). The upper copper electrode (9) is now free to be removed and the sample (20) is placed over the lower copper electrode (10). The two electrodes are made of copper with highly polished surfaces and have screws for fixing connection wires. The lower electrode (10) is welded to long copper rode (21) in which another soft iron rode (24) is fixed tightly. A stiff spring (11) hangs up the lower electrode (10) and its rods and keeps the iron rod inside a copper cylinder with thick mouth $(12,22)$. The whole system is fully software controlled. The power control signals and the data signals are delivered from and to a computer system through an USB multi-channel interface (National Instruments 
model NI6009). The motor (16) is activated by a running signal from the interface through a high power relay. The gear box (15) reduces the motor speed and increases its torque, then the cylindrical gear (14) rotates the disk gear (1) which, in turn rotates the screwed rod (4) through a big fixed nut (5). The result of these rotations is vertical down displacement of the rod (4) causing compression on the sample. This compression is conducted to the spring (11) which is contracted (strained) by a distance that is linearly proportional to the exerted force on the sample. The soft iron rod (24) moves down a distance exactly equals the strain of the spring.

A small transformer excites the primary coil (25) that is wounded around the copper cylinder (22) using 3 volts and 60 $\mathrm{Hz}$. This alternating low voltage produces alternating magnetic field in the soft iron movable core (24). The movement of the core (24) transfers the magnetic lines of force to cross the secondary coil (26) and hence an induced voltage appears between terminals of the secondary coil (26). The induced voltage on coil (26) is proportional to the depth of rod (24) inside the coil due to the continuous increase in the number of coil turns (26) that are crossed by the iron core. Finally, voltage drop across terminals of coil (26) is directly proportional to the force acted on the sample and this voltage can be easily calibrated to obtain the force directly. The voltage across coil (26) is delivered to an analog input channel of the interface while the calibration relation is included in the controlling software so that the force is measured immediately. Introducing the sample surface area to the software enables the software to calculate and save the net pressure applied on the sample.

Electrodes (9) and (10) are connected to DC-power supply of constant output voltage $\mathrm{V}_{\mathrm{cc}}$ as shown in the circuit diagram [1].

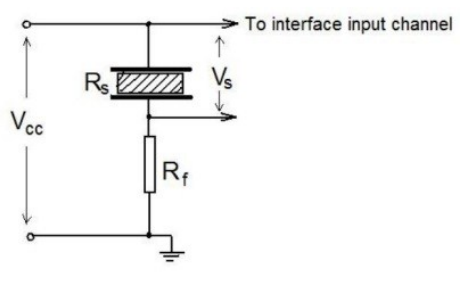

Figure 2: design of the circuit diagram 1

An interface input channel receives the voltage $\left(V_{s}\right)$ across the sample, while the current $\left(\mathrm{I}_{\mathrm{s}}\right)$ flows in the sample is calculated using the following relation:

$$
I_{s}=\frac{V_{c c}-V_{s}}{R_{f}}
$$

Where $\mathrm{R}_{\mathrm{f}}$ is a constant resistance $(25 \pm 0.01 \square)$. Hence the sample resistance $R_{s}$ could be calculated one by one, with sample rate of $10 \mathrm{~Hz}$ from the relation:

$$
R_{S}=\frac{V_{s}}{I_{s}}=\frac{V_{s} \cdot R_{f}}{V_{c c}-V_{s}}
$$

Upper and lower limits of some measured variables are hardware selected, which are:
1 - The DC motor speed, where the motor (16) is powered from external power supply across a potentiometer. The motor speed is controlled manually using a potentiometer.

2 - The force applied to the sample and the limits of the force are controlled by the stiffness of the spring (11) and it's allowed maximum compression strain. This strain is limited by the length of the window (13) that is grooved in the cylinder (12), and the steel stopper (23). The limits of applied force can be adjusted by exchange the spring only, where the maximum compressive strain is fixed.

3 - The applied pressure on the sample, where limits of applied pressure are controlled not only by limits of applied force but also by surface area of the sample. The diameter of the electrodes (9) and (10) is one inch $(25.4 \mathrm{~mm})$, hence the maximum surface area for the sample is $506.7 \mathrm{~mm}^{2}$ and the smallest sample will be approximately $4 \times 4=16 \mathrm{~mm}^{2}$. This means that the upper limit of the applied pressure can be over 30 times the lower limit according to the selected sample area. This technique is easy and fast for changing rang of applied pressure, since changing the force limits requires changing the spring and hence recalibrate the instrument.

4 - The total applied voltage $\mathrm{V}_{\mathrm{cc}}$, where its value is taken constant through a run; it can be adjusted from the source. The total applied voltage may be alternating with selectable frequency if the capacitive effect of the sample under variable pressure was a task.

\section{TECHNICAL MEASURES FOR HIGHER PERFORMANCE:}

Some measurements have been considered to enhance the performance of the instrument;

1 - Pressure release: as the force acted on the sample reaches its maximum value, which is pre-identified through software, as the motor (16) is stopped and reverses the rotation direction. The steel stopper (23) is introduced in the instrument to force the motor to stop in case of software or computer failure. During the pressure release, the software continues data recording. This feature helps to detect the pressure - resistance hysteresis, which provides important knowledge about the electro - mechanical behaviour of the tested QTC samples.

2 - Best insulation: Teflon thick plates are used as very good insulator to prevent leakage of electric currents through movable or fixed parts of the instrument. General view of the instrument shows that the instrument is divided into two main parts, above each other separated by the sample only.

3 - Smoothing the motion: A small lubricated steel ball (29) is placed between the rotating screw bar (4) and the upper electrode (9) to prevent rotation of the electrode and to keep the force directed downward for always.

\section{TESTING THE INSTRUMENT}


This instrument is designed and assembled specially to measure the electro-mechanical properties of any quantum tunnelling composites (QTC). In order to test the efficiency and performance of this instrument, it was essential to prepare a QTC samples and measure their physical properties.

\section{1 - QTC samples preparation:}

The common structure of a QTC is polymer-metal composite, or in more general consideration is polymer - conducting solid composite. The widely used polymer for QTCs is silicon rubbers. They consist of poly-dimethyl siloxane (PDMS), curing agent, fillers and additives. After application, they are cross-linked by contact with atmospheric moisture releasing by-products in the process. Cross-linking starts with the formation of a skin on the surface of the applied silicon rubber and gradually works its way into the compound. Their outstanding weathering and aging resistance is the result of their special chemical properties. Commercial silicon rubber is used in this work as a host polymer. Graphite was selected as a conducting component.

Commercial graphite rods were crushed manually in a mortar as trial to produce fine particles (flakes) with acute tips that may be equivalent to the spikes of other metals. Silicon rubber was mixed manually with different amount of graphite flakes to produces composite with different silicon rubber to graphite ratios $(1: 0.75,1: 1,1: 1.5,1: 1.75$ and 1:2) as a weight fraction. The produced mixtures were then casted into Teflon mould and left overnight for curing. Afterwards, the composites were cut into discs with dimensions of $24.5 \mathrm{~mm}$ diameter and $2 \mathrm{~mm}$ thickness.

\section{Scanning electron microscopy (SEM):}

Specimens were sputter-coated with gold and examined using a JEOL $6400 \mathrm{SEM}$ at an accelerating voltage of $30 \mathrm{kV}$ in secondary electron mode.

The SEM micrographs show the size and shapes of the used graphite powder (see figure 3 ).

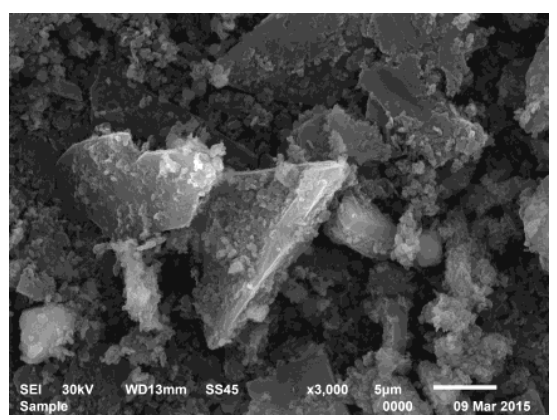

(A)

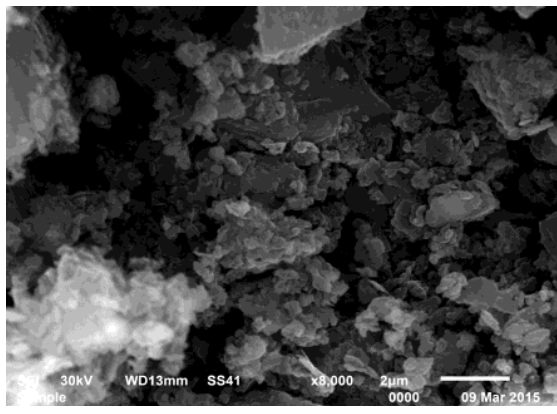

(B)

Figure 3: SEM micrographs for the used graphite powder with two different magnifications.

Graphite flakes has range of sizes, ranged from submicron to several microns $(\sim 10 \mu \mathrm{m})$. This wide range of sizes was attributed to the preparation method (i.e. manual crushing). It can be also observed that the flakes have irregular geometries with sharp tips which is essential for preparation of QTC.

These images show the sharp tips of the graphite fragments in both large and tiny fragments.

\section{2 - Repeatability and accuracy of the instrument:}

Two factors are indirectly affecting the electro - mechanical response of some QTCs; the rate of pressing and the maximum pressure acting on the sample.

a) The rate of pressing a sample:

The rate of pressing is directly proportional to the number of revolutions per minute (RPM) of the driving DC motor. The motor RPM is controlled manually from a power potentiometer. As the motor is excited by certain voltage, it rotates with a fixed speed unless its applied voltage is changed to another required value to acquire new speed, and so on. Many samples can be measured at the same pressing rate as long as the motor voltage is kept constant. By this feature, the electro - mechanical properties of different samples can be compared irrespectively to the effect of pressing rate. Figure 4 shows the determination method of the pressing rate, where the pressure is recorded as function of time through a complete cycle of pressing and releasing the sample, then the pressing rate was determined as $(\mathrm{dp} / \mathrm{dt})$ gradient. Figure 4 shows also that the pressure, acted on the sample is exactly linear with time, especially at higher speeds of the motor. At low speed, the little non-linearity is due to the mechanism of changing the motor speed. The RPM is reduced by lowering the driving power of the motor, while the mechanical resistance (stiffness of the spring (11) in Figure 1) still linear. The little shift from the zero point on the pressure axis in figure 4 was suggested to be due to the initial low pressure acted on the sample by the weight of the upper thick electrode (9) in figure 1. Figure 4 shows also the accuracy of the mechanical system in verifying the selected maximum pressure regardless to the selected pressing rate.

b) b. Selection of maximum applied pressure: 
Most of QTC applications are sensing applications, where the sensing property of the sensor should be reversible. In the case of sensors for mechanical variables (force, pressure, strain,....etc), the applied stress to the sensing material should not exceed the yield point of the material. Therefore, the maximum pressure or force that applied on the sample during measurements should be adjustable either by the measuring hardware or the controlling software.

At this point, it should be differentiated between two maxima of pressure. The first is the maximum pressure can be applied by the instrument, which is determined by the stiffness and length of the spring (11) (see Figure 1). The second maximum pressure is that selected to be applied on the sample during a specific run. Therefore, the maximum pressure through a run is always less than the maximum pressure by the instrument. Figure 5 shows the pressure applied to a sample versus time for different runs each at different selected maximum pressure.

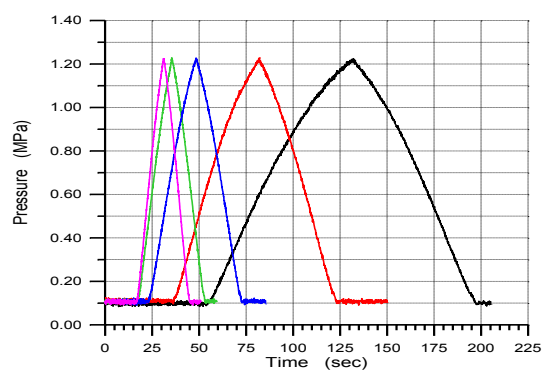

Figure 4: The applied pressure versus time at Different pressing rates and the same selected maximum pressure.

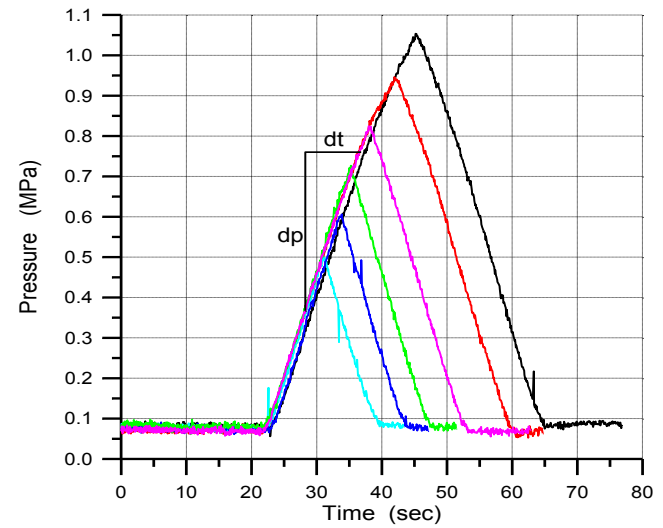

Figure 5: Applied pressure against time at different maximum pressure

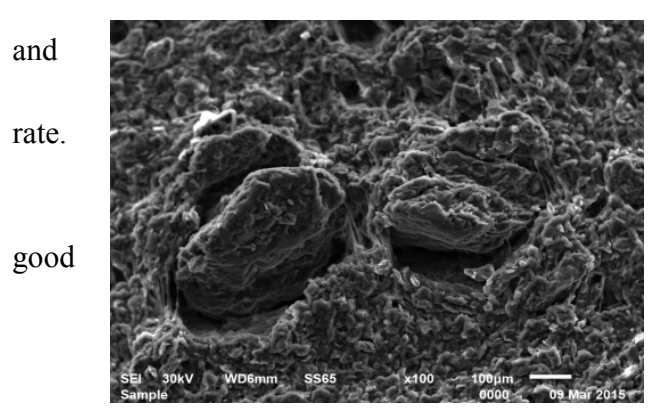

values the same pressing

Figure 5 shows

repeatability of the pressing rate regardless the selected maximum pressure. In the current instrument, the maximum pressure during a run is software selected.

Now the instrument is qualified to be used satisfactorily for characterizing the electro-mechanical properties of quantum tunnelling composites

\section{CASE STUDY:}

Two kinds of piezoresistive composites should be distinguished based on the conduction mechanism through the dispersed phase. The former composite type, also known as pressure conductive rubber, exhibits a variation of the electrical conduction in response to a mechanical deformation, as a consequence of the contact change among the conductive particles. To describe the formation of conductive paths under external load, originated by intimately contacted particles, different percolation models have been proposed $[4,5]$.

Generally, these models fail below the percolation threshold where they predict that the composite is an insulator. The latter piezoresistive composite type (known as quantum tunnelling composite, QTC) show a conduction mechanism that can be well represented by field assisted Fowler-Nordheim tunnelling model, where the filler particles are well separated and being fully coated with the insulating polymeric matrix [6].

The prepared composites were used in the following study. Figure 6 represents SEM micrographs for silicon rubber/graphite composites showing the fully coating of graphite flakes with silicon rubber. Therefore, there is no potential for percolation to occur.

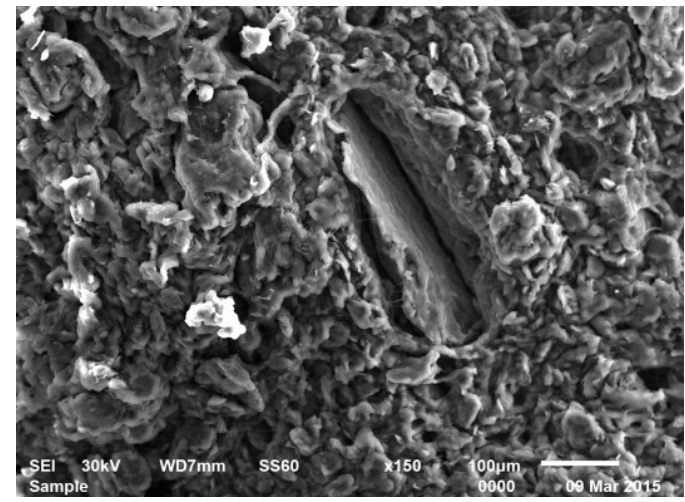

(b)

a) Figure 6: SEM micrographs for silicon rubber/graphite composites with different compositions; (a) $1: 2$ and(b) $1: 1.5$.Graphite particles are imbedded and fully coated with the polymer matrix where

The automatic dynamic measurement of the QTC was used to record the sample current Is during the continuous increase in the applied pressure at different pressing rates. Figure 7 illustrates change in Is against applied pressure. Some remarkable notes can be drawn from this curve:

a) The sample current and resistance are independent on the pressing rate. This statement appears in contrast with a well- 
known viscoelastic concept, where the material shows more stiffness at faster rates of load application and vice versa. In fact, the current case is somewhat differs than the viscoelastic concept because the time interval between pressing and releasing is significantly shorter (see figure 5) than the mechanical relaxation time of the sample. In the current study, the relaxation process was measured statically and results can be seen in figure 8 . Figure 8 shows that increasing the graphite content (filler concentration) causing increase in the stiffness of the sample and accordingly, the mechanical relaxation time decreased.

b) Figure 7 also demonstrates good accuracy and repeatability in measurement of the sample current.

c) The little fluctuations in Figure 7 was ascribed to nonhomogenous distribution of graphite flakes within the specimens.

For some applications, the decay of sample current at removing of pressure is as important as the current growth during pressing. Therefore, it was necessary to record the current hysteresis (loading and unloading). The suggested low cost setup able to record the current flows in the sample during both stages of loading and unloading. From Figure 9, turn point of the pressure can be seen and highlighted inside the blue circle. It is clear that the current dropped exactly at the turn point of pressure and soon its value is recovered. Finally, it decayed as fast as the pressure decreased. The question arises here is; what is the source of charges that are compensated the blockage of charge flow due to the sudden increase of resistance at the turn point? The increase of current at the tipping point may be due to the capacitance of the sample between conductive electrodes. Chen et al. [7] cleared that the contact resistance of the electrodes $\mathrm{R}_{\mathrm{e}}$ is negligible and then the sample may be considered as a parallel connection of a variable resistance and a variable capacitor (see Diagram 2).Therefore, it was essential to confirm this deduction by measuring the effect of capacitive feature of the sample on the measured sample current under different loads.

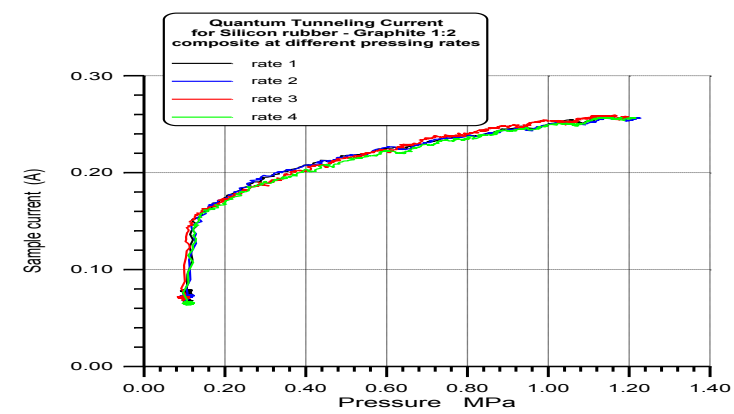

Figure 7 : Sample current as function of the applied pressure for 1: 2 rubber : graphite composite.

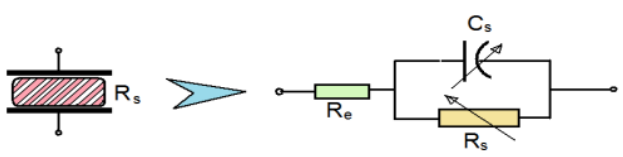

Diagram 2: The equivalent circuit diagram of the sample

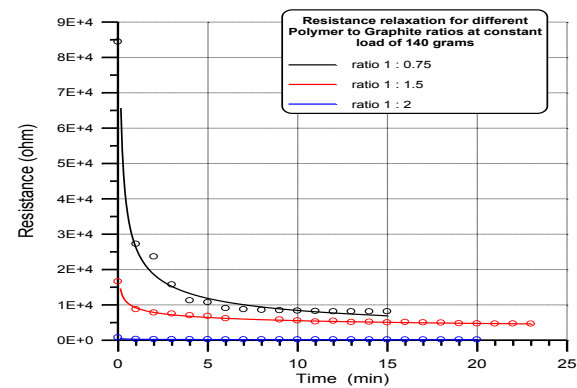

Figure 8: The relaxation of sample resistance for silicon rubber/graphite with different compositions under a constant load of 140 grams

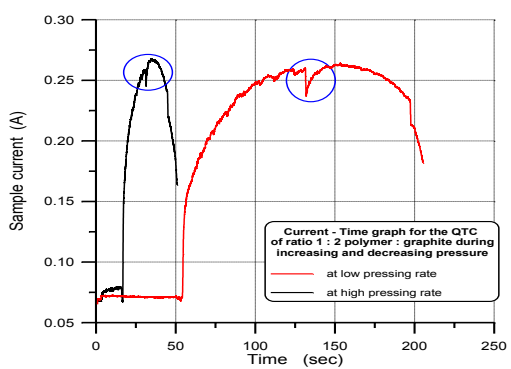

Figure 9: Current vs. time graph for the QTC of ratio $1: 2$ composite during loading and unloading stages at high and low rates showing the turn point.

Figure 10 shows the capacitance of the produced QTC with ratio of $1: 1$ at different applied loads. The capacitance was measured by a digital multi-meter while the sample was under static load. The readings were recorded after complete relaxation of the sample. As the load increased from 0.3 to 2.6 $\mathrm{kg}$, the capacitance of the composite increased gradually (approximately linearly) from $25 \mu \mathrm{F}$ to reach $200 \mu \mathrm{F}$ at the load of $2.6 \mathrm{~kg}$. Figure 11 also demonstrates the change in capacitance against applied load for composites with higher rubber to graphite ratios. Comparing the load ranges and the corresponding change of capacitance in Figures 10 and 11, It can be deduced that composites with higher graphite concentrations possess higher sensitivity for capacitance change under loading, even if they have higher thickness. This was attributed to the existence of high amounts of conducting particles (graphite). Figures $10 \& 11$ show also that at zero load (un-deformation) the samples showed very low capacitance which started to increase by applying a load. This means that the QTC has a potential to be used as a pressure variable condenser. 


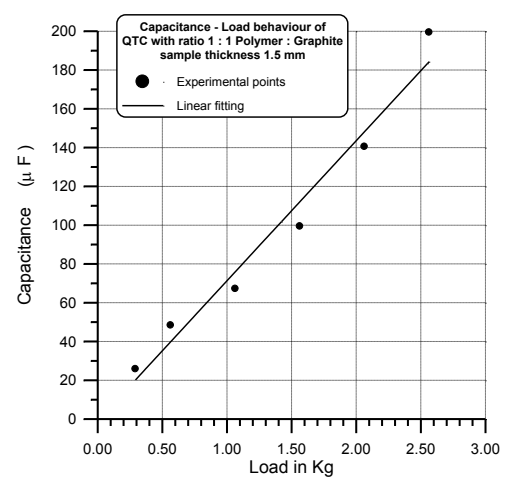

Figure 10: Change in the specimen capacitance versus load for composites of $1: 1$ ratio and $1.5 \mathrm{~mm}$ initial thickness.

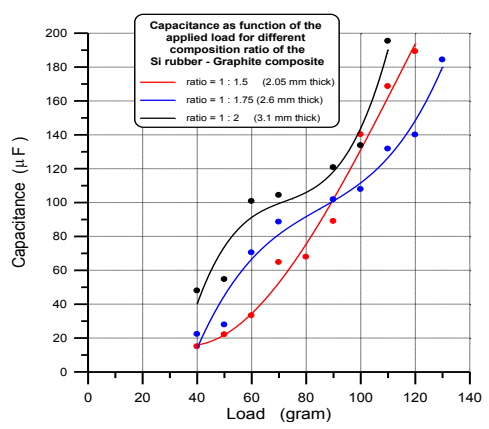

Figure 11: Capacitance as function of the applied load for different composition ratio of the silicon rubber - graphite composites

Changes in sample capacitance $\left(\mathrm{C}_{\mathrm{s}}\right)$ means changes in its reactance $\left(\mathrm{X}_{\mathrm{s}}\right)$, where $\mathrm{X}_{\mathrm{s}}=1 / \square \mathrm{C}_{\mathrm{s}}=1 / 2 \square \mathrm{fC}_{\mathrm{s}}(\square \square$ is the angular frequency and $\mathrm{f}$ is the frequency of applied voltage). Consequently, the sample current should also depend on the frequency of applied voltage. Change in sample currents against frequency for composites two different graphite concentrations at different loads is shown in Figure 12 and 13. These graphs clarify the current dependence on $\mathrm{f}$ and $\mathrm{X}_{\mathrm{s}}$ separately. The position of a curve belongs to the effect of $X_{s}$ by the applied load, while the trend of each curve belongs to the effect of the frequency of the applied voltage. Figure 13 shows that at higher graphite concentrations, the effect of different loads is minimized while the effect of frequency is sharpen up.

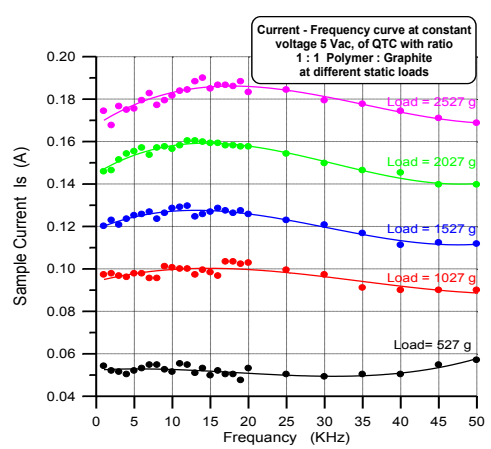

Figure 12: Sample current versus frequency of applied voltage to QTC with 1:1 ratio at different mechanical loads.

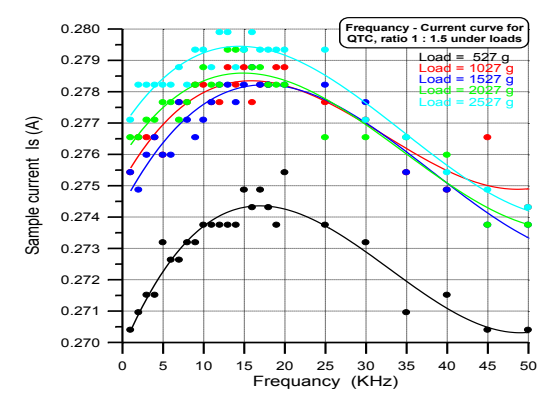

Figure 13: change in sample current against frequency of applied voltage to $1: 1.5$ composite at different mechanical loads.

\section{CONCLUSION:}

Carbon materials such as graphite, carbon black (CB) and carbon nano-tubes (CNTs) are widely used as fillers in conductive polymer composites due to low density and high conductivity. Many studies have recently demonstrated that polymers especially soft elastomers incorporated with these conducting fillers could show significant piezoresistive performance [8]. A low cost technique was introduced in this study to carry out most of the required measurements in order to characterize the electro-mechanical properties of QTC using both DC and AC applied voltage. The suggested setup provided a stream of data presenting many physical parameters with satisfactorily accuracy and repeatability. This data stream should be very helpful guide for scientists whom interested in developing QTC and modelling the process of quantum tunnelling in metal based composites.

\section{REFERENCES:}

1 -www.nanolab.unimore.it

2 - D. Bloor, A. Graham, E. J. Williams, P. J. Laughlin, and D. Lussey, "M etal-polymer composite with nanostructured filler particles and amplified physical properties," Applied Physics Letters, vol. 88, no. 10,pp. 102103-102103-3, Mar. (2006).

3 - YWR Amarasinghe1, AL Kulasekera, TGP Priyadarshana, Seventh International Conference on Sensing Technology,(2013)

4 - Hussain M., Choa Y.-H., Niihara K. "Conductive rubber materials for

pressure sensors." Journal of Materials Science Letters, 20, pp. 525- 527 (2001)

5 - Ding T., Wang L., Wang P. "Changes in electrical resistance of carbon-

black-filledsilicone rubber composite during compression. Journal of Polymer Science Part B:Polymer Physics, 45, pp. 2700-2706(2007)

6 - S. Stefano, G. Canavese, F. Cosiansi, M. Cocuzza, “An innovative Cupper-PDMS Piezoresistive composite for 
flexible tactile sensor" The

15thEUROPEAN CONFERENCE ON COMPOSITE

MATERIALS,

Venice, Italy, 24-28 June (2012)

7 - Qian Chen, Yingying Sun, Ying Wang, Hongbin Cheng, Qing-Ming

Wang, "ZnO nano-wires-polyimide nano-composite piezoresistive strain

sensor",Sensors and Actuators A 190, pp. 161-167(2013)

8 -WentingCai, Ying Huang, Dayue Wang, Caixia Liu, Yugang

Zhang, "Piezoresistive Behaviour of Graphene Nanoplatelets/ Carbon

Black/ Silicone Rubber Nano-composite” J. APPL. POLYM.

SCI.

(2013), DOI: 10.1002/APP.39778 